Supporting Information

\title{
Bi-Phasic Ag-In-Ga Embedded Elastomer inks for Digitally Printed, Ultra-Stretchable, Multi-layer
}

\section{Electronics}

Pedro Alhais Lopes ${ }^{l} \dot{\dagger}$, Daniel Félix Fernandes ${ }^{I} \dot{\dagger}$, André F. Silva ${ }^{1}$, Daniel Green

Marques $^{l}$, Aníbal T. de Almeida ${ }^{l}$, Carmel Majidi ${ }^{2 *}$, Mahmoud Tavakoli ${ }^{1 *}$

1. Institute of Systems and Robotics, Department of Electrical Engineering, University of Coimbra, Coimbra, 3030-290, Portugal

2. Integrated Soft Materials Lab, Department of Mechanical Engineering, Carnegie Mellon University, Pittsburgh, PA 15213, USA

$\dagger$ First Co-Authors

*Corresponding Authors Email:

Prof. C. Majidi: cmajidi@andrew.cmu.edu,

Prof M. Tavakoli:mahmoud@isr.uc.pt 
Figure S1. shows dimensions of the dogbone used for performing the electromechanical coupling characterization.

Figure S2. shows the EDS analysis of the Microstructure, in which three zones are distinguishable. EGaln droplet covered by Gallium Oxide, Ag flakes that are distributed all over the sample, and the newly formed AgIn 2 particles, that are mainly present at the borders of the Gallium Oxide. Wt\%, and At\% calculation of these particles, confirms the 2:1 atomic percentage between $\mathrm{In}$ and $\mathrm{Ag}$, thus $\mathrm{AgI} \mathrm{n}_{2}$. Note that the relatively large EGaln droplet visible in this figure, is due to breaking of one (or several), smaller EGaln droplets during preparation of the crosssection. Otherwise, these droplets are in the order of $<10 \mu \mathrm{m}$ of size, as can be seen in Fig. S8.

Figure S3. is a amplified image of Figure S2, in which areas of accumulation of each of the 3 microstructures are shown.

Figure S4. is another elemental analysis map.

Figure S5. compares the microstructure of two samples, one pristine, and another one poststretch, and a computer vision analysis of the sizes of the particles. No significant difference is seen for the pre-stretch, and post-stretch sample. Also, another sample that was left under $100 \%$ strain overnight was analyzed, which as well did not show any significant change in the microstructure.

Figure S6. The thickness of printed samples, that was used in conductivity analysis.

Figure S7 and Table S1, show the study performed on the Ag-SIS inks without LM, to obtain the best composition. This was done mainly to have a fair comparison between the best ink without EGaln, and our AgInGa ink.

Figure S8. shows another cross-section image, in which two EGaln droplets are identified and measured.

Video S1. shows printing of a sample circuit using the extrusion printer setup, over a medical dressing, Tegaderm, and the non-smearing behavior of the ink after drying.

Video S2. another example of printing a circuit.

Video S3. a fully passive, battery-less circuit printed over medical adhesive, interfaced with the human skin, and is powered through electromagnetic coupling (Near-Field Harvesting). The circuit sends its ID to the mobile phone, which opens the SPM-ISR website. The multi-layer construction of the coil contributes to a better efficiency, in energy harvesting.

Video S4 and S5 show printing of a strain gauge, and its function over the human wrist, for monitoring the bending angle.

Video S6. is another example of fully passive printed stretchable circuit, that is powered using the energy harvested by the printed antenna, through RFID (Far field harvesting).

Video S7. Shows an example of a circuit with interfacing LEDs, under repetitive $30 \%$ strain.

Video S8. Shows a smearing test comparing AgInGaSIS ink and EGaln LM using strips of PDMS and Eco Flex. 

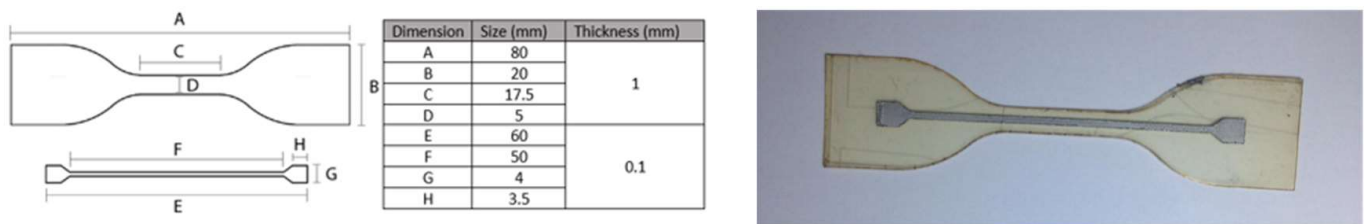

Figure S1. Dimensions of the dogbone, and the printed ink for electrical and electromechanical characterization. We used latex as the substrate for all EM tests.

$\begin{array}{lccl}\text { Element } & \text { Wt } \% & \text { At } \% & \\ \text { C K } & 1.82 & 10.36 & \text { Z1: Ga-In large body } \\ \text { GaL } & 80.93 & 79.37 & \\ \text { InL } & 17.25 & 10.27 & \\ & & & \\ \text { C K } & 2.19 & 16.60 & \text { Z2: Ag-In or Agln } 2 \\ \text { GaL } & 8.32 & 10.87 & \text { Ga nearby } \\ \text { AgL } & 29.00 & 24.50 & \\ \text { InL } & 60.50 & 48.03 & \\ & & & \\ \text { C K } & 3.18 & 22.78 & \text { Z3: Silver Flake (flat) } \\ \text { AgL } & 96.82 & 77.22 & \\ & & & \\ \text { C K } & 1.85 & 14.98 & \text { Z4: Ag-In or Ag-In2 } \\ \text { AgL } & 31.58 & 28.52 & \sim \text { Same percentage } \\ \text { InL } & 66.58 & 56.50 & \text { of Z2 } \\ & & & \end{array}$

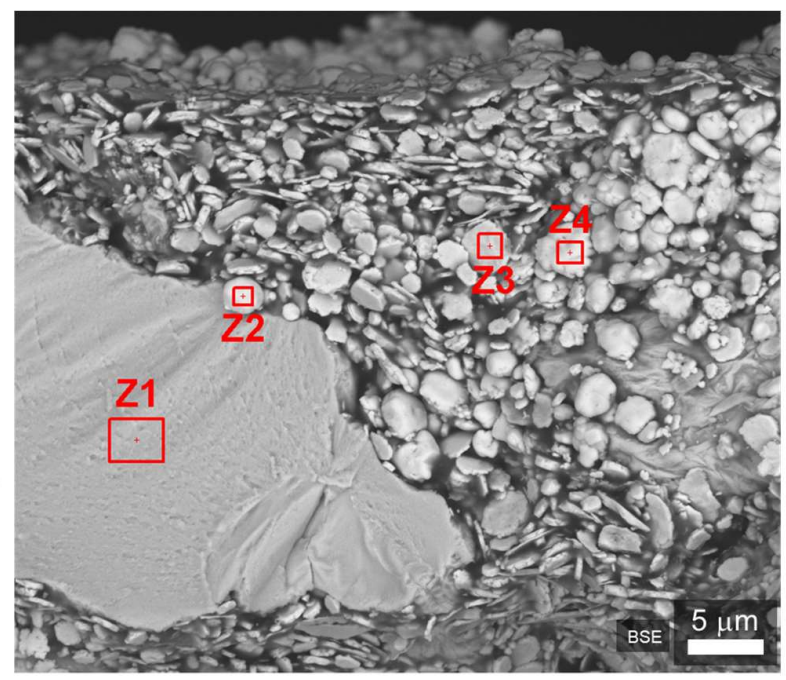

Figure S2. EDS Analysis of the 3 distinct microstructures. Z1: Near Eutectic Galn drop teared down during cross section preparation. Z2\&Z4: Ag-In or Ag- $\ln _{2}$, with the same \% of $\mathrm{Ag}$ and In. Z3: Ag flakes 


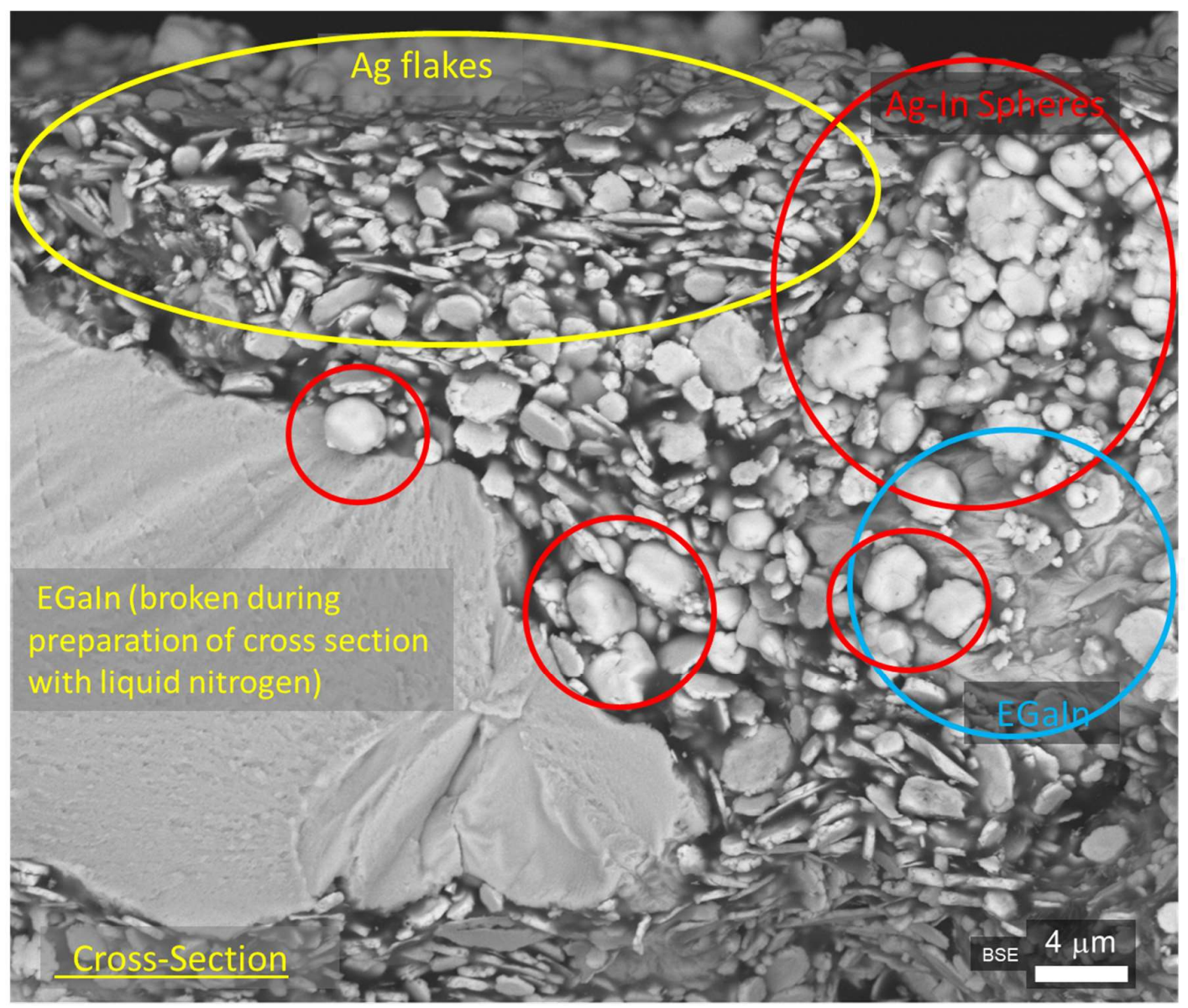

Figure S3. EDS Analysis of the 3 distinct microstructures. Z1: EGaln drop teared down during cross section preparation. Z2\&Z4: Ag-In or Ag-In ${ }_{2}$, with the same \% of Ag and In. Z3: Ag flakes

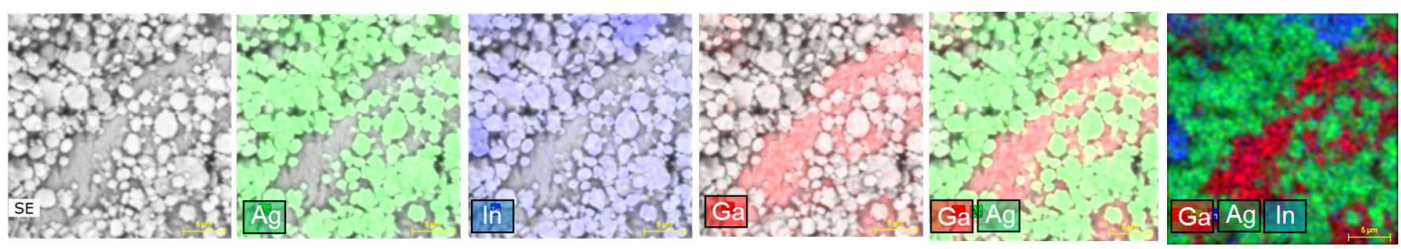

Figure S4 SEM and EDS analysis of a sample. Ag and Ga are present in separate areas. In and Ag have co-presence in the same zones. 


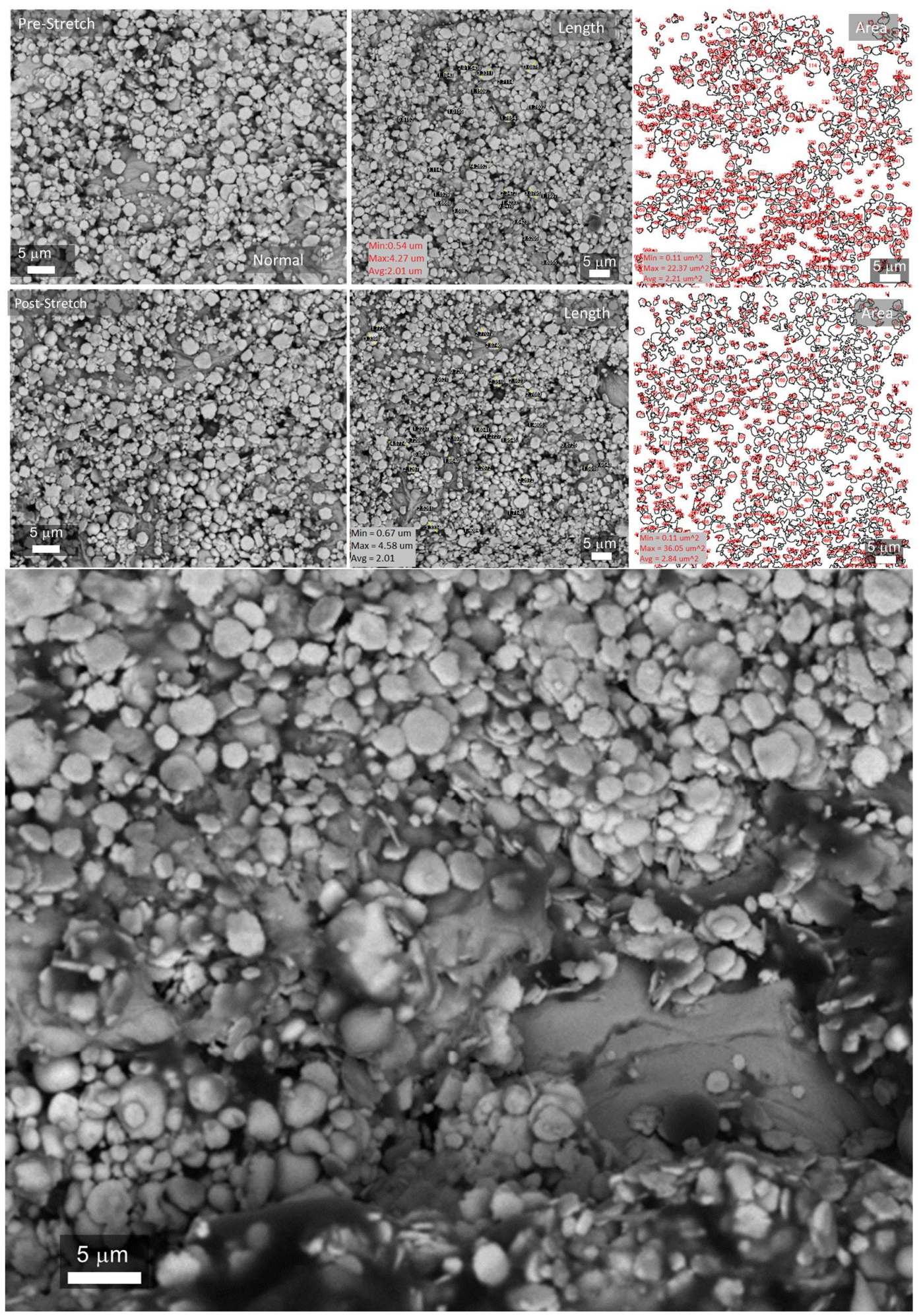

Figure S5. SEM BSE analysis of a sample Pre-stretch and Post-Stretch. No significant change can be observed. Below: A sample of the ink which was left under $100 \%$ strain overnight. 


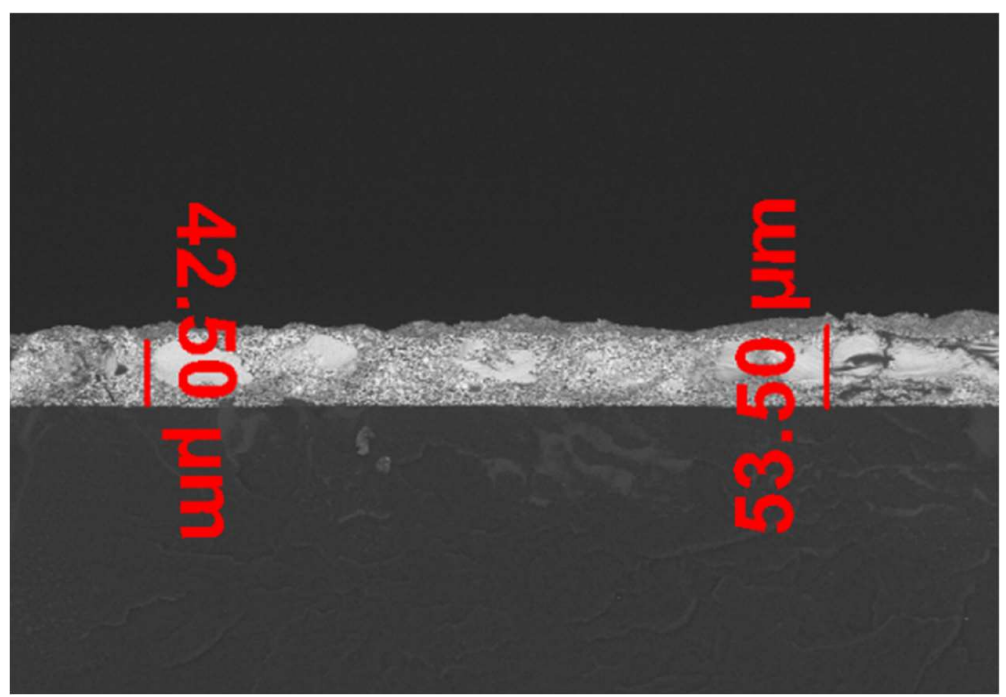

Figure S6. Cross sectional analysis of the printed samples that are used for estimation of the conductance, shows thickness varying from $37 \mu \mathrm{m}$ to $53 \mu \mathrm{m}$ in different locations.

\section{AgSIS composite:}

We performed an initial optimization of AgSIS composite, to reach to the best combination of stretchability, and conductivity, before comparison with the Ag-In-Ga-SIS composite. The AgSIS composite with $89 \mathrm{wt} \%$ of metal content, which results in the best combination of conductivity, and stretchability for the Ag-SIS composite without liquid metal (Figure S7, and Table S1). Reducing further the amount of SIS to less than $10 \%$ of the total composition, makes the sample even more brittle, reducing the maximum strain to less than $50 \%$ of strain. Figure 57 , and Table S1, summarize the behavior of different AgSIS samples.

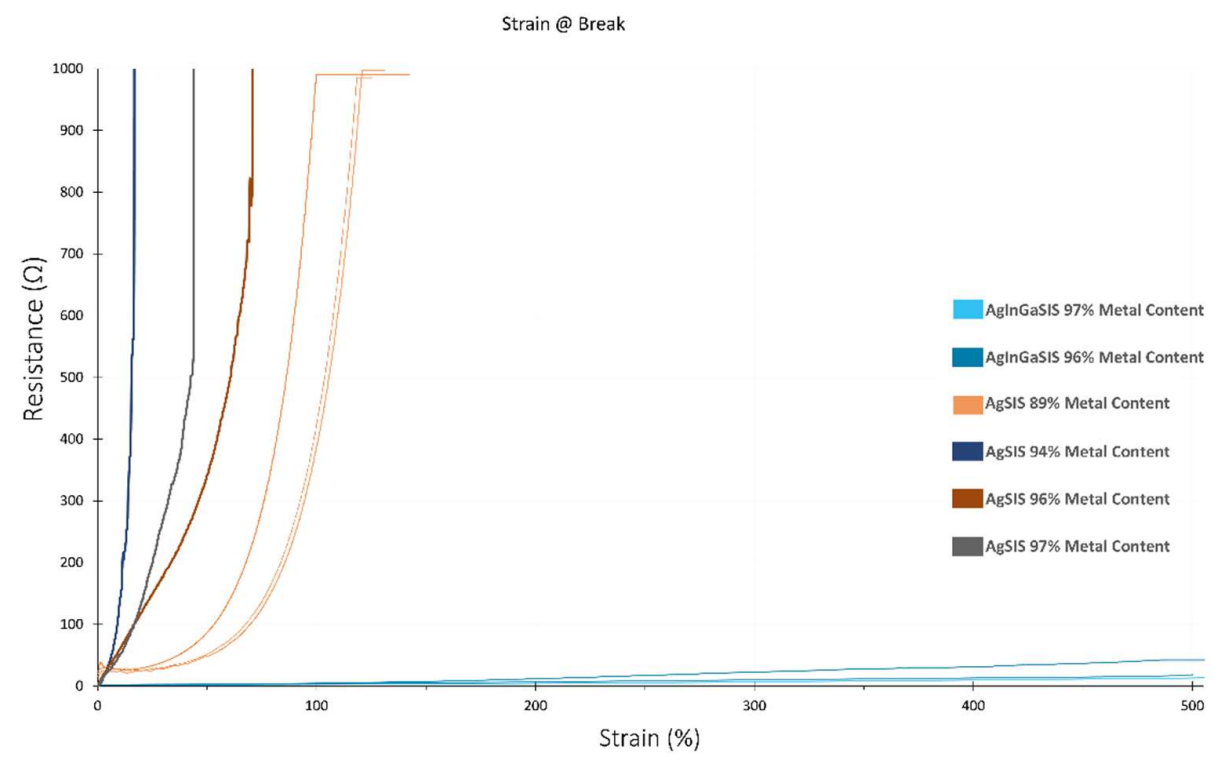

Figure S7. Electromechanical coupling behaviour of AgSIS samples with 89-97 wt\% metal content. The $89 \%$ was found to be the composition that withstands the maximum strain of up to $140 \%$. 


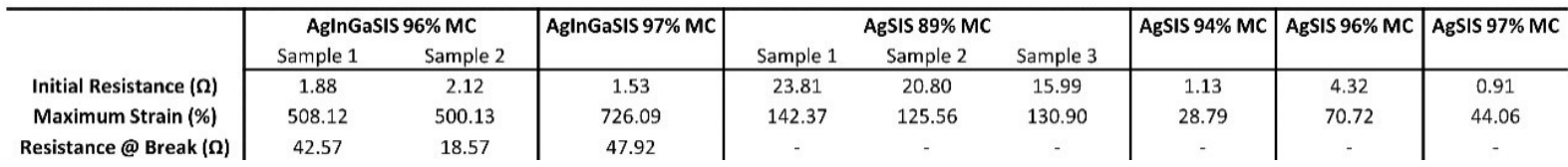

Table S1. Comparison of electrical conductivity, and maximum strain of AgSIS samples made with $89-97 w t \%$ of $\mathrm{Ag}$ flake content.

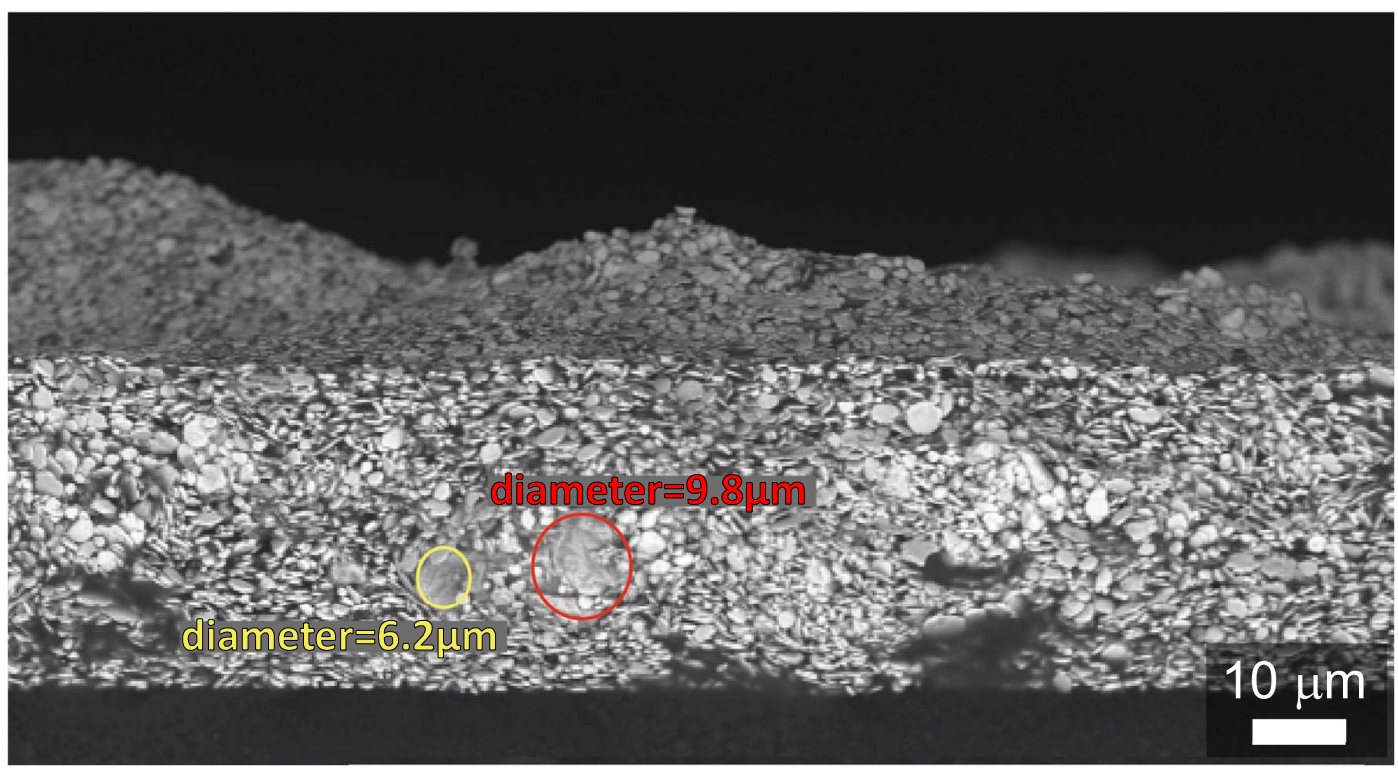

Figure S8. Diameter of EGaln droplets ranging from 5-10 $\mu \mathrm{m}$. 